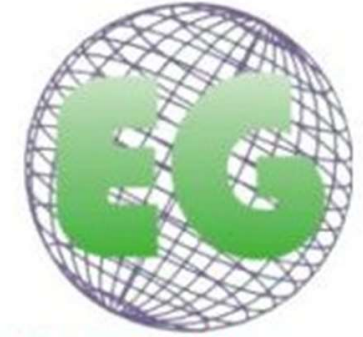

ISSN 1695-6141 N'57

\title{
Efecto de un programa de paciente experto en insuficiencia cardiaca
} Effect of an expert patient program in heart failure

\author{
Diana Marcela Achury-Saldaña ${ }^{1}$ \\ Laura Restrepo ${ }^{2}$ \\ María Kamila Munar ${ }^{2}$ \\ Indira Rodríguez ${ }^{2}$ \\ María Camila Cely ${ }^{2}$ \\ Natalia Abril ${ }^{2}$ \\ Lina Toledo ${ }^{2}$
}

1 Pontificia Universidad Javeriana. Profesora asociada de la Facultad de Enfermería y coordinadora del
Semillero GESCCA: Gestores de Cuidado Cardiovascular. Bogotá. Colombia. dachury@javeriana.edu.co

2 Pontificia Universidad Javeriana. Facultad de Enfermería, Semillero GESCCA: Gestores de Cuidado Cardiovascular. Bogotá. Colombia.

\section{http://dx.doi.org/10.6018/eglobal.19.1.361801}

Recibido: 5/02/2019

Aceptado: 21/02/2019

\section{RESUMEN:}

Introducción: Una estrategia que ha mostrado ser efectiva para promover el autocuidado en los pacientes con falla cardiaca es la formación de diadas y grupos que brinden apoyo de pares para favorecer el logro de metas individuales.

Objetivo general: Determinar el efecto de un programa de paciente experto en falla cardiaca en los conocimientos relacionados con la adherencia al tratamiento y en la satisfacción.

Método: Se realizó un estudio piloto descriptivo de corte transversal en pacientes con diagnósticos de falla cardiaca en una institución de cuarto nivel. El tamaño de la muestra correspondió a toda la población con los criterios de restricción (N: 20 sujetos). Se implementó un programa de paciente experto durante 6 meses y se midieron los desenlaces de nivel de conocimientos y satisfacción. Para la recolección de la información se utilizó un test de conocimientos sobre adherencia al tratamiento y una encuesta de satisfacción. La información se procesó a través del programa IBM SPSS Statistics 23.0, y se realizó un análisis descriptivo con distribución de frecuencias absolutas y relativas.

Resultados: Más del 95\% de los pacientes mostró mejoría significativa en el nivel de conocimientos y el $85 \%$ refirió el nivel de satisfacción global más alto (muy satisfechos), reflejado en la dimensión de fidelización y efectividad.

Conclusiones: El programa del paciente experto en insuficiencia cardiaca se constituye en una intervención costo-efectiva que proporciona capacidades para ayudar a otros pacientes a adquirir autoconfianza y habilidades en el manejo de su condición de salud.

Palabras clave: Insuficiencia cardiaca; paciente experto; autocontrol. 


\begin{abstract}
:
Introduction: One of the strategies that has shown improvement in self-care for patients with heart failure is the formation of dyads and groups to provide peer support and achieve individual goals.

General objective: To determine the effect of an expert patient program on heart failure related to the knowledge about adherence to treatment and satisfaction.

Method: A sectional-descriptive pilot study was conducted in patients with heart failure diagnoses in a fourth-level institution. The sample size represents the entire population with the restriction criteria ( $\mathrm{N}$ : 20 subjects). An expert patient program was implemented for 6 months and the measured outcomes were level of knowledge and satisfaction. Data was gathered from a knowledge test on adherence to treatment and a satisfaction poll. Information was processed with IBM SPSS Statistics 23.0. The distribution of absolute and relative frequencies was performed by descriptive analysis.

Results: More than $95 \%$ of patients showed improvement in the level of knowledge and $85 \%$ reported a high global level of satisfaction (very satisfied), reflected in the dimension of loyalty and effectiveness.

Conclusions: The expert patient in heart failure program is a cost-effective intervention, which provides capabilities to help other patients gain self-confidence and skills to manage their health condition.
\end{abstract}

Keywords: Heart failure; expert patient; self-control.

\title{
INTRODUCCIÓN
}

Actualmente, la falla cardíaca (FC) se considera una de las principales enfermedades no transmisibles que generan un gran impacto en la población. Se estima que el aumento en su incidencia esté relacionado con el envejecimiento y el incremento de los factores de riesgo cardiovasculares. A nivel mundial, afecta a 26 millones de personas. Así mismo, representa la tercera causa de muerte en los países desarrollados ${ }^{(1)}$.

En Colombia, el $2.3 \%$ de la población presenta FC y en 2013 se reportó un aumento del $40,8 \%$ de reingresos hospitalarios a causa descompensaciones ${ }^{(2)}$, lo que genera costos elevados para el sistema de salud y compromete la calidad de vida de los pacientes y su familia (3). Resulta necesario, entonces, desarrollar estrategias adecuadas para mitigar esta problemática, orientadas a promover la participación del paciente en el cuidado y toma de decisiones, con el fin de lograr un adecuado nivel de autocuidado y adherencia al tratamiento farmacológico y no-farmacológico (4).

La Organización Mundial de Salud (OMS) señala en el nuevo Modelo de cuidados crónicos que se debe desarrollar en los pacientes la capacidad de tomar de decisiones sobre su estado de salud y generar cambios conductuales en sus estilos de vida para promover el autocuidado. Con este propósito, el profesional de la salud debe integrar al cuidado tradicional diferentes estrategias educativas lideradas por los pacientes ${ }^{(5)}$.

Una estrategia que ha mostrado ser efectiva es la formación de "pacientes expertos", donde las personas que padecen una enfermedad crónica reciben orientación y se forman para desarrollar habilidades concernientes al manejo de su condición, y que les permiten incluso ayudar a otros pacientes a adquirir autoconfianza. Se busca establecer una conexión entre dos o más personas que posean características comunes, como la presencia de una enfermedad, para favorecer el logro de metas individuales o grupales. Los pacientes adquieren un rol activo y protagónico, y los profesionales de la salud un rol pasivo u observador, un acompañamiento que puede actuar como reconductor en el caso que fuera necesario ${ }^{(6)}$.

Una de las iniciativas que responde a esta estrategia es el denominado "Chronic Disease Self-Management Program". Este proyecto nace en 1990 en la Universidad 
de Stanford con el propósito de transferir conocimiento y habilidades relacionadas con los cuidados específicos de la enfermedad para aumentar el autocuidado. La universidad cuenta con programas dirigidos a pacientes con artritis, diabetes, personas con $\mathrm{VIH}$, dolor crónico y un taller en el que se abarcan diversas enfermedades crónicas (CDSMP) ${ }^{(7)}$. Progresivamente, la metodología fue impulsando la extensión del programa a nivel mundial: Reino Unido, Universidad de los Pacientes (UAB), la Fundación Josep Laporte (UAB), Barcelona, Andalucía, Cataluña, entre otros, los cuales pretenden mejorar la comprensión y la toma de conciencia de la enfermedad crónica por parte de los ciudadanos. Implementado en pacientes con falla cardiaca y evaluando su nivel de satisfacción, se resalta un resultado de satisfacción de 4.6 sobre 5 , lo que se refleja como adopción de un papel auto responsable por parte de los pacientes participantes y, a la vez, puede ser clínica y económicamente efectivo ${ }^{(8)}$.

La implementación de este programa debe estar enmarcada en su efectividad. Autores como González (9), Griffiths (10) y Holden (11) recomiendan evaluar su impacto por medio del nivel de conocimiento y la satisfacción, como determinantes para generar autonomía, corresponsabilidad y empoderamiento del paciente. Así mismo, señalan que su puesta en práctica permitirá ir cambiando el paradigma del modelo biomédico que enmarca el cuidado, lo que resultará en un mejor complemento a la labor de educación realizada por los profesionales de la salud.

En Colombia, solo se cuenta con un programa de paciente experto liderado por la Fundación Santa Fe de Bogotá, el cual ofrece estrategias educativas a los pacientes con enfermedades crónicas y a sus familiares, permitiendo que estos asuman compromisos con el cuidado de la salud y la prevención de enfermedades. Sin embargo, este programa no es específico para la población de pacientes con FC. En el país aún son escasos este tipo de programas que, aunque están contemplados en la guía técnica Buenas prácticas para la seguridad del paciente en la atención en salud del Ministerio de la Protección Social de la República de Colombia (12) y pretenden fortalecer el autocuidado de los pacientes, no han alcanzado el impacto y la relevancia que requieren. Esto se debe a que se continúa manejando un sistema de salud predominantemente paternalista, y los médicos y enfermeras son aún quienes manejan de manera exclusiva la información; se encargan de la educación al paciente y dejan de lado la posibilidad de utilizar la modalidad de "programa paciente experto" como herramienta para educar y mejorar las cifras de morbimortalidad actuales por FC.

De igual manera, se identifican renuencias por parte de los profesionales de salud a la hora de diseñar este tipo de programas, cuando perciben que pueden perder su papel protagónico en la educación (13). Por lo anterior, se evidencia un vacío de conocimiento y la necesidad de involucrar el nuevo perfil del paciente experto con falla cardiaca en las instituciones de salud, además de evaluar su efecto a corto y largo plazo. El alcance de esta investigación fue la evaluación a corto plazo.

\section{OBJETIVO}

Determinar el efecto de un programa de paciente experto en falla cardiaca en los conocimientos relacionados con la adherencia al tratamiento y en la satisfacción. 


\section{MÉTODO}

\section{Diseño, población y tamaño de la muestra}

Se realizó un estudio piloto descriptivo de corte transversal en pacientes con diagnósticos de FC que asistieron a consulta ambulatoria en una institución de cuarto nivel. Los criterios de inclusión fueron: pacientes hombres o mujeres mayores de 18 años, con diagnóstico de falla cardiaca que se encuentren vinculados a un programa de falla cardiaca. El tamaño de la muestra correspondió a toda la población con los criterios de restricción ( $\mathrm{N}$ : 20 sujetos), mediante un muestreo no probabilístico. Los pacientes con clase funcional IV y que se encontraban en tratamiento de paliación y no podían participar del programa fueron excluidos del estudio.

\section{Intervención}

La intervención consistió en el diseño y aplicación del programa de paciente experto. La primera etapa consistió en seleccionar los pacientes que liderarían el programa y formarían a sus pares. Los pacientes seleccionados como expertos debían poseer características como: adecuadas capacidades comunicativas y una adecuada adherencia al tratamiento. La formación de estos pacientes líderes se realizó durante 4 sesiones, con una periodicidad de una vez por semana y una duración de 2 horas cada una. El contenido de las sesiones educativas en el proceso de formación del paciente se orientó a metodologías de enseñanza en adultos, y el manejo del tratamiento farmacológico y no farmacológico a partir de la experiencia vivida. Una vez finalizadas las sesiones, se realizó una evaluación al paciente experto a partir de un juego de rol (role playing). La segunda etapa se orientó al proceso de formación de pares por parte de los expertos. Para este fin, se realizó un cribado de pacientes que quisieran participar, se formó un grupo de 20 pacientes, y el experto realizó 6 sesiones educativas durante 6 meses con una periodicidad mensual. La metodología utilizada en las sesiones educativas fue reflexiva, a partir de preguntas orientadoras sobre la experiencia vivida en el cambio de comportamientos frente al tratamiento farmacológico y no farmacológico. El rol activo durante el programa fue del paciente experto y los profesionales de enfermería tuvieron un rol pasivo de observadores; sólo intervenían si era necesario. Al finalizar la última sesión se aplicó el test de conocimiento y la encuesta de satisfacción.

\section{Desenlace principal}

El nivel de satisfacción y los cambios en los conocimientos relacionados con la adherencia al tratamiento farmacológico y no farmacológico.

\section{Instrumento}

Se diseñó un test de conocimiento de 10 preguntas con escala de repuesta de selección múltiple para evaluar los aspectos de adherencia al tratamiento no farmacológico contemplados en la Guía europea sobre el diagnóstico y tratamiento de la insuficiencia cardiaca aguda y crónica ${ }^{(14)}$, tales como la dieta, el control de peso, la actividad física, los signos de alarma y la toma de medicamentos y líquidos. Con el objeto de evaluar la satisfacción, se realizó una encuesta con 13 preguntas, a partir de los referentes de Hall y Dornan ${ }^{(15)}$, destinada a medir tres dimensiones: calidad del programa, competencia técnica y fidelización. Cuenta con una escala tipo Likert con 
cinco opciones de respuesta, donde uno es el valor más bajo (muy insatisfecho) y cinco el más alto (muy satisfecho). El test de conocimiento se aplicó antes y al finalizar el programa de paciente experto, y la encuesta de satisfacción al finalizar. Se ejecutó una prueba piloto con 5 pacientes y una revisión por 2 expertos profesionales con el propósito de examinar la claridad y comprensión de la encuesta y el test.

\section{Procedimiento}

\section{Plan de análisis}

La información se procesó a través del programa IBM SPSS Statistics 23.0 de 2014. Se realizó un análisis descriptivo con distribución de frecuencias absolutas y relativas.

\section{Aspectos éticos}

El estudio cumplió los estándares de las normas científicas, técnicas y administrativas para la investigación en salud, Resolución 008430 de 1993 del Ministerio de Salud de la República de Colombia. Se consideró un estudio de riesgo mínimo y, adicionalmente, se tuvieron en cuenta los principios éticos relacionados al respeto y autonomía, beneficencia - no maleficencia, justicia y confidencialidad. El estudio se adhirió a normas éticas nacionales o internacionales para la protección de los derechos de los participantes de la investigación. Finalmente, se incluye la participación voluntaria, la confidencialidad de la información y el consentimiento informado por parte de los participantes al estudio.

\section{RESULTADOS}

Tabla 1: Variables sociodemográficas

\begin{tabular}{|l|l|c|c|}
\hline \multicolumn{2}{|c|}{ Característica } & N. $^{\circ}$ & Porcentaje \\
\hline \multirow{5}{*}{ Género } & Mujer & 9 & $45 \%$ \\
\cline { 2 - 4 } & Hombre & 11 & $55 \%$ \\
\hline \multirow{5}{*}{ Edad } & $21-40$ años & 2 & $10 \%$ \\
\hline \multirow{5}{*}{ Escolaridad } & $41-60$ años & 8 & $40 \%$ \\
\cline { 2 - 4 } & Mayor a 61 años & 10 & $50 \%$ \\
\hline \multirow{5}{*}{ Ocupación } & Primaria & 5 & $25 \%$ \\
\cline { 2 - 4 } & Bachillerato & 11 & $55 \%$ \\
\cline { 2 - 4 } & Universidad & 4 & $20 \%$ \\
\hline \multirow{5}{*}{ diempo de } & Hogar & 9 & $45 \%$ \\
\cline { 2 - 4 } & Empleado & 3 & $15 \%$ \\
\cline { 2 - 4 } & Independiente & 7 & $40 \%$ \\
\hline \multirow{3}{*}{ Cuidador } & $<5$ años & 10 & $35 \%$ \\
\cline { 2 - 4 } & De 5-10 años & 20 & $50 \%$ \\
\cline { 2 - 4 } & Más de 10 años & 0 & $15 \%$ \\
\hline
\end{tabular}

Fuente: resultados del estudio 
Se observa que la mayoría de los pacientes eran mujeres con edad promedio entre 41- 61 años, con escolaridad bachillerato, cuya ocupación era el hogar, seguida de independiente, con un tiempo diagnóstico de 5 a 10 años. Todos cuentan con un cuidador (Tabla 1).

Tabla 2: Resultados del test de conocimientos antes y después de la aplicación del programa paciente experto

\begin{tabular}{|c|c|c|c|c|c|}
\hline \multicolumn{3}{|c|}{ Antes de la aplicación del programa } & \multicolumn{3}{|c|}{ Después de la aplicación del programa } \\
\hline \multicolumn{6}{|c|}{ Dieta y control del sodio } \\
\hline $\mathbf{N} .^{\circ}$ & Porcentaje & Respuesta & $\mathbf{N}^{\circ}$ & Porcentaje & Respuesta \\
\hline 7 & $35 \%$ & Conoce & 20 & $100 \%$ & Conoce \\
\hline 13 & $65 \%$ & No conoce & 0 & $0 \%$ & No conoce \\
\hline \begin{tabular}{l|l} 
Total & 20 \\
\end{tabular} & $100 \%$ & & \begin{tabular}{|l|l|} 
Total & 20 \\
\end{tabular} & $100 \%$ & \\
\hline \multicolumn{6}{|c|}{ Actividad física } \\
\hline N. ${ }^{\circ}$ & Porcentaje & Respuesta & $\mathbf{N}^{\circ}$ & Porcentaje & Respuesta \\
\hline 8 & $40 \%$ & Conoce & 19 & $95 \%$ & Conoce \\
\hline 11 & $60 \%$ & No conoce & 1 & $5 \%$ & No conoce \\
\hline \begin{tabular}{l|l} 
Total & 20 \\
\end{tabular} & $100 \%$ & & \begin{tabular}{|l|l|} 
Total & 20 \\
\end{tabular} & $100 \%$ & \\
\hline \multicolumn{6}{|c|}{ Signos de alarma } \\
\hline N. ${ }^{\circ}$ & Porcentaje & Respuesta & $\mathbf{N}^{\circ}$ & Porcentaje & Respuesta \\
\hline 7 & $35 \%$ & Conoce & 19 & $95 \%$ & Conoce \\
\hline 13 & $65 \%$ & No conoce & 1 & $5 \%$ & No conoce \\
\hline \begin{tabular}{l|l} 
Total & 20 \\
\end{tabular} & $100 \%$ & & \begin{tabular}{|l|l|} 
Total & 20 \\
\end{tabular} & $100 \%$ & \\
\hline \multicolumn{6}{|c|}{ TOMA DE MEDICAMENTOS } \\
\hline $\mathbf{N}^{\circ}$ & Porcentaje & Respuesta & $\mathbf{N}^{\circ}$ & Porcentaje & Respuesta \\
\hline 6 & $30 \%$ & Conoce & 20 & $100 \%$ & Conoce \\
\hline 14 & $70 \%$ & No conoce & 0 & $0 \%$ & No conoce \\
\hline \begin{tabular}{l|l} 
Total & 20 \\
\end{tabular} & $100 \%$ & & \begin{tabular}{|l|l|} 
Total & 20 \\
\end{tabular} & $100 \%$ & \\
\hline \multicolumn{6}{|c|}{ LIQUIDOS } \\
\hline $\mathbf{N}^{\circ}$ & Porcentaje & Respuesta & $\mathbf{N}^{\circ}$ & Porcentaje & Respuesta \\
\hline 7 & $35 \%$ & Conoce & 19 & $95 \%$ & Conoce \\
\hline 13 & $60 \%$ & No conoce & 1 & $5 \%$ & No conoce \\
\hline \begin{tabular}{l|l} 
Total & 20
\end{tabular} & $100 \%$ & & \begin{tabular}{|l|l|} 
Total & 20 \\
\end{tabular} & $100 \%$ & \\
\hline
\end{tabular}

Fuente: resultados del estudio

Antes del programa de pacientes expertos se encontró que la mayoría de los participantes no conocían de forma clara y precisa las recomendaciones relacionadas con los medicamentos $(70 \%)$, dieta $(60 \%)$, signos de alarma $(65 \%)$ y control de líquidos (60\%). Al finalizar las 6 sesiones con periodicidad mensual, se evidenció una mejoría significativa en los conocimientos (95-100\%) (Tabla 2).

Tabla 3: Satisfacción global de los pacientes con el programa paciente experto

\begin{tabular}{|c|c|c|}
\hline \multicolumn{3}{|c|}{ Satisfacción global } \\
\hline Nivel de satisfacción & N. $^{\circ}$ & Porcentaje \\
\hline Muy satisfecho & & $85 \%$ \\
\hline Satisfecho & 17 & $5 \%$ \\
\hline Aceptable & 1 & $10 \%$ \\
\hline Insatisfecho & 2 & $0 \%$ \\
\hline Muy insatisfecho & 0 & $0 \%$ \\
\hline Total & 0 & $100 \%$ \\
\hline
\end{tabular}

Fuente: resultados del estudio 
Figura 1: Satisfacción global de los pacientes con el programa paciente experto

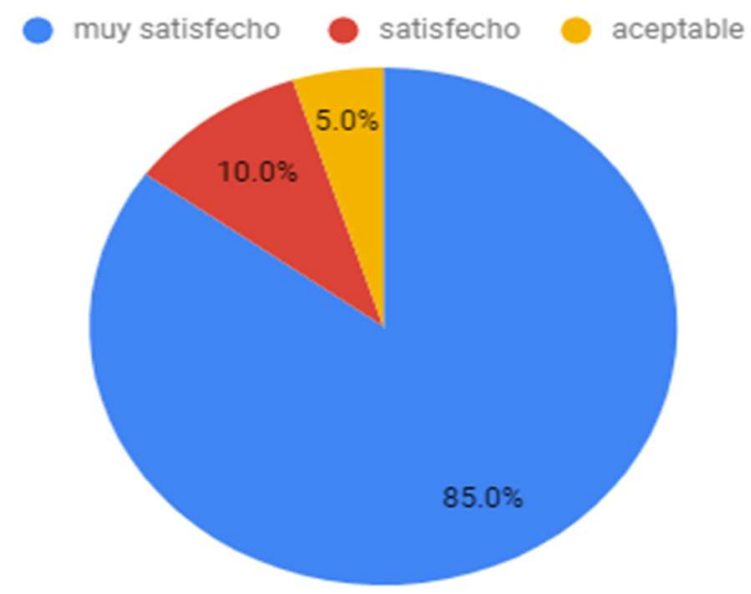

Fuente: resultados del estudio

La clasificación global de satisfacción muestra que la mayoría de los pacientes participantes se encontraban muy satisfechos con lo aprendido y alcanzado en el programa (Tabla 3; Figura 1).

Tabla 4: Descripción de dimensiones de satisfacción del programa paciente experto

\begin{tabular}{|c|c|c|c|}
\hline \multicolumn{4}{|c|}{ Dimensión calidad del programa } \\
\hline \multicolumn{2}{|c|}{ N. ${ }^{\circ}$} & Porcentaje & Nivel de satisfacción \\
\hline \multicolumn{2}{|c|}{16} & $80 \%$ & Muy satisfecho \\
\hline \multicolumn{2}{|c|}{3} & $15 \%$ & Satisfecho \\
\hline \multicolumn{2}{|c|}{1} & $5 \%$ & Aceptable \\
\hline \multicolumn{2}{|c|}{0} & $0 \%$ & Insatisfecho \\
\hline \multicolumn{2}{|c|}{0} & $0 \%$ & Muy insatisfecho \\
\hline Total & 20 & $100 \%$ & \\
\hline \multicolumn{4}{|c|}{ Dimensión competencia técnica } \\
\hline \multicolumn{2}{|c|}{ N. ${ }^{\circ}$} & Porcentaje & Nivel de satisfacción \\
\hline \multicolumn{2}{|c|}{14} & $70 \%$ & Muy satisfecho \\
\hline \multicolumn{2}{|c|}{4} & $20 \%$ & Satisfecho \\
\hline \multicolumn{2}{|c|}{2} & $10 \%$ & Aceptable \\
\hline \multicolumn{2}{|c|}{0} & $0 \%$ & Insatisfecho \\
\hline \multicolumn{2}{|c|}{0} & $0 \%$ & Muy insatisfecho \\
\hline Total & 20 & $100 \%$ & \\
\hline \multicolumn{4}{|c|}{ Dimensión fidelización } \\
\hline \multicolumn{2}{|c|}{ N..$^{\circ}$} & Porcentaje & Nivel de satisfacción \\
\hline \multicolumn{2}{|c|}{18} & $90 \%$ & Muy satisfecho \\
\hline \multicolumn{2}{|c|}{2} & $10 \%$ & Satisfecho \\
\hline \multicolumn{2}{|c|}{0} & $0 \%$ & Aceptable \\
\hline \multicolumn{2}{|c|}{0} & $0 \%$ & Insatisfecho \\
\hline \multicolumn{2}{|c|}{0} & $0 \%$ & Muy insatisfecho \\
\hline Total & 20 & $100 \%$ & \\
\hline
\end{tabular}




\begin{tabular}{|c|c|c|c|}
\hline \multicolumn{4}{|c|}{ Dimensión efectividad del programa } \\
\hline & & Porcentaje & Nivel de satisfacción \\
\hline & & $100 \%$ & Muy satisfecho \\
\hline & & $0 \%$ & Satisfecho \\
\hline & & $0 \%$ & Aceptable \\
\hline & & $0 \%$ & Insatisfecho \\
\hline & & $0 \%$ & Muy insatisfecho \\
\hline Total & 20 & $100 \%$ & \\
\hline
\end{tabular}

Fuente: resultados del estudio

Figura 2: dimensiones de satisfacción del programa paciente experto

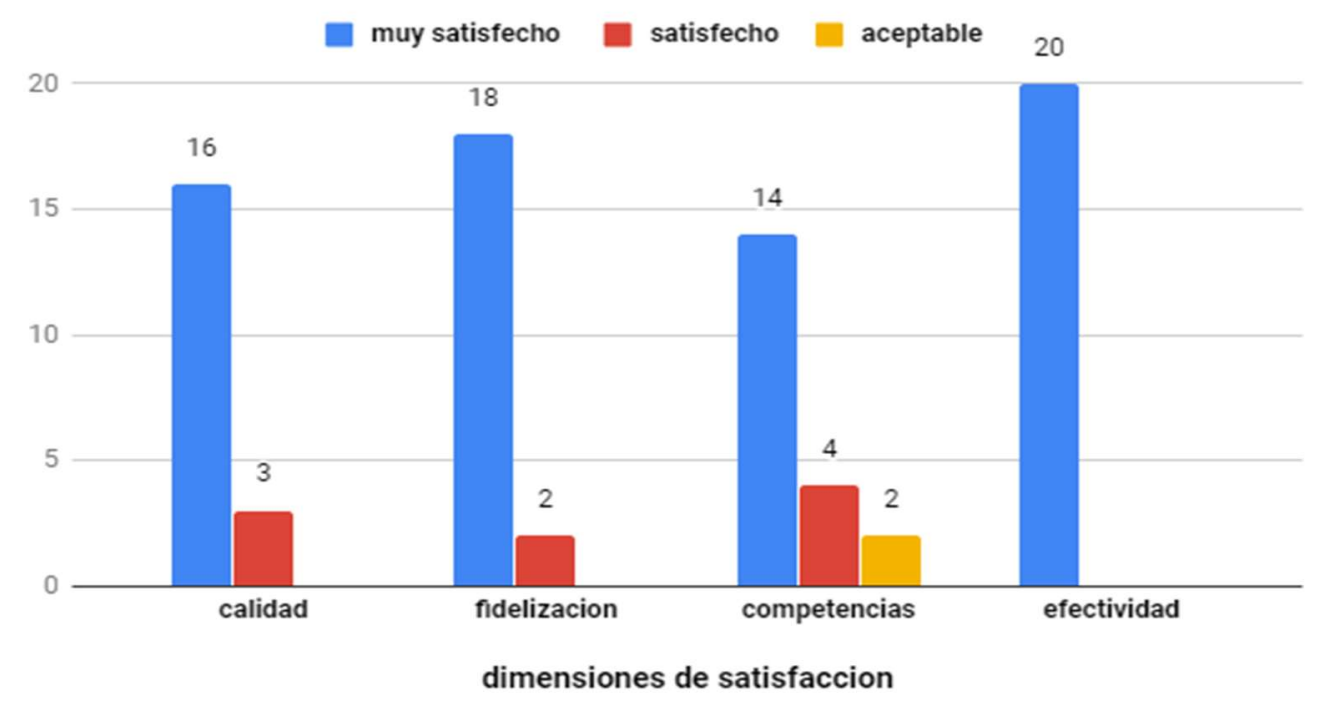

Fuente: resultados del estudio

En la dimensión de calidad del programa, referida al tipo y precisión de la información, la claridad en sus interrogantes y la metodología utilizada se encontró que el $80 \%$ de los participantes estaban muy satisfechos. Con respecto a las competencias técnicas, entendidas como la capacidad de los pacientes expertos en el proceso enseñanzaaprendizaje, se evidencio que los participantes estaban muy satisfechos en su mayor porcentaje $(70 \%)$. En la dimensión de fidelización, que hace referencia a las expectativas y recomendación del programa, se encontró un $90 \%$ de pacientes muy satisfechos. Por último, en la dimensión efectividad, que hace referencia al impacto del programa reflejado en los cambios significativos que han tenido en sus estilos de vida y en su autocuidado a partir de los aprendido y vivido en las sesiones, el $100 \%$ de los participantes estaban muy satisfechos (Tabla 4; Figura 2).

\section{DISCUSIÓN}

Los resultados muestran que el efecto del programa de paciente experto generó un cambio muy importante en el grado de conocimiento y nivel de satisfacción. Con relación al grado de conocimiento, se evidenció que la mayoría de los pacientes al finalizar las sesiones del programa mejoraron de forma significativa sus conocimientos relacionados con el tratamiento farmacológico y no farmacológico, como los medicamentos y la dieta, seguidos del reconocimiento de signos de alarma, actividad 
física y control de líquidos. Las guías mundiales de diagnóstico y manejo de FC sugieren que el paciente debe recibir educación para incrementar el nivel de conocimiento sobre el manejo adecuado de su tratamiento, con el propósito de generar cambios a largo plazo que le permitan tener un buen control de su enfermedad y evitar posibles descompensaciones ${ }^{(16)}$.

Autores como Salvadó-Hernández ${ }^{(17)}$ señalan que existe un reto en la búsqueda de estrategias que logren incrementar la adherencia al tratamiento no farmacológico desde los servicios de salud, dado que numerosos estudios han demostrado que los medicamentos por sí solos no son suficientes para el control de la enfermedad o sus complicaciones. Por tal motivo, se hace necesario promover la educación, no sólo con enfoque individual sino grupal, en donde los pacientes puedan tener un rol protagónico y puedan formar otros pares a partir del ejemplo y las estrategias de cambio de sus comportamientos en el autocuidado.

Barrios (18) señala que el conocimiento es un conjunto de información almacenada mediante la experiencia o el aprendizaje (a posteriori) o a través de la introspección (a priori). Existen muchas maneras de transmitir dicho conocimiento; uno de los métodos más usados es el explícito, en el que se transmite la información a los sujetos por medio de una comunicación formal. Dicho proceso puede ser brindado tanto por el personal sanitario como por pacientes expertos, quienes han tenido un recorrido teórico y vivencial, por lo tanto, cuentan con las bases y sustentos necesarios para educar o transmitir su información a los pacientes aprendices. Éstos estarán más abiertos a adherirse al conocimiento que viene de alguien con quien se sienten identificados.

También es importante destacar que el profesional de enfermería tiene un rol fundamental en este proceso, puesto que es el encargado de complementar y apoyar al paciente experto en caso de que sea necesario. Ambas partes generan un complemento importante: por una parte, el paciente experto aporta sus experiencias y consejos (tips) para el día a día. Por otra parte, el profesional aporta conocimientos técnicos y específicos que son esenciales para lograr el empoderamiento de los pacientes. Lo descrito se puede confirmar con autores como Molina et al. (19), quienes afirman que la información que es transmitida por un paciente experto llega de forma más directa al paciente par, ya que se realiza desde un nivel similar al suyo, con una experiencia parecida y utilizando su mismo lenguaje. Esto, a su vez, ayuda a evitar la actitud derrotista de algunos pacientes frente al personal sanitario y a que afloren las dudas, dificultades y preocupaciones que los pacientes tienen con relación a su enfermedad.

Los resultados positivos alcanzados en el nivel de conocimientos en este estudio coinciden con la investigación de González A ${ }^{(20)}$, en donde se evidenciaron mejoras en el autocuidado de los pacientes que fueron parte del programa de paciente experto, esto gracias a las vivencias que los pacientes expertos compartieron con los pacientes aprendices. Esto promovió cambios positivos en los hábitos y estilos de vida de los pacientes, por medio de acciones que les permitieron reconocer, tratar y gestionar sus problemas de salud de forma autónoma.

Por otra parte, algunas variables sociodemográficas caracterizadas en este estudio, como "ser mujer" y un "nivel medio-alto de escolaridad", pudieron favorecer la mejoría en el conocimiento. Al hacer una revisión de la literatura, Barreiro ${ }^{(21)}$ mostró que el mayor grado de desconocimiento sobre la enfermedad crónica se asoció con un bajo 
nivel de estudios. También se podría afirmar que el hecho de ser mujer otorga a los pacientes habilidades para aprender un poco más rápido, porque su pensamiento se tipifica en red y la información bajo este esquema es permanentemente totalizada.

En cuanto al nivel global de satisfacción, se encontró que los pacientes participantes se encontraban en su mayoría muy satisfechos con el programa, lo cual revela el cumplimiento de los principales objetivos planteados. Se proporcionó a los participantes una oportunidad para empoderarse de todo el conocimiento correspondiente a la falla cardiaca, no solo a nivel teórico sino práctico, incluyendo aspectos de carácter emocional, a fin de mejorar su calidad de vida a mediano y largo plazo. Un nivel alto de satisfacción está influenciado, pues los pacientes se sienten identificados con otros individuos que han vivido la enfermedad y son capaces de orientar a otros en el autocuidado y la autorregulación de su salud. De esta manera, se combinan los cuidados ofrecidos por los profesionales, basados en la evidencia, con los propios de la vivencia y la experiencia.

Pesquera y López-López (22) señalan en su artículo lo enunciado por Prieto M, directora de la primera escuela de pacientes en España: este tipo de proyectos, además de mejorar la calidad de vida de los pacientes, disminuye el número de visitas al médico. Además, señala que la iniciativa parte del concepto de que cuando se conoce bien la enfermedad, y cuando los pacientes reciben información entre iguales, las enfermedades avanzan más despacio, disminuyen los efectos secundarios y aumenta la satisfacción. Por lo tanto, la dependencia del sistema sanitario es menor y la adherencia terapéutica mayor, resultados equiparables a los obtenidos en el presente estudio.

Por su parte, en el estudio llevado a cabo por Torres-Gonzáles y León-Manco ${ }^{(23)}$ se documenta que el nivel de satisfacción es un concepto muy importante:

"[...] por la subjetividad que lo acompaña, ya que está enfocada más hacia las percepciones y actitudes de la persona que hacía criterios concretos y objetivos. También permite evaluar la perspectiva que tienen los pacientes de los servicios que le son prestados y puede servir como predictor de la calidad del cuidado que se les está ofreciendo".

Santana et al. ${ }^{24)}$ recomiendan escuchar a los pacientes sobre el cuidado entregado, ya que es una oportunidad de construcción de un indicador de resultado que orienta a los gestores sobre los posibles caminos decisorios de transformaciones e innovaciones.

La satisfacción es un concepto multidimensional en el que se evalúan de manera individual distintos aspectos relacionados con la atención entregada. Las dimensiones mejor calificadas por los participantes del presente estudio fueron la efectividad y la fidelización. Según Gómez-Ramírez et al. ${ }^{(25)}$, la percepción de satisfacción es definitiva para reconocer la calidad de un servicio. Por lo tanto, como ratifica Ampuero $^{(26)}$, se puede afirmar que existe una relación significativa entre la calidad del servicio y la fidelización y efectividad del paciente. Mientras mejor sea la calidad de atención que se le brinde al paciente, se presentan más oportunidades para lograr la fidelización con los pacientes. En este sentido, las instituciones de salud deben ofertar servicios de calidad que garanticen la fidelización del paciente; es decir, que la persona tenga cierta preferencia y recomiende la institución a otros. 
De acuerdo con Díaz y Gil (27), la efectividad es una dimensión que cobra gran importancia en la satisfacción. Si los usuarios identifican un cambio en su autocuidado y el cumplimiento de los objetivos planificados, reconocerán los beneficios en su estado de salud. La efectividad alcanzada del programa de paciente experto del presente estudio se logró gracias a la metodología de aprendizaje vivencial o experiencial utilizada, puesto que permitió mejorar los conocimientos y aumentar la satisfacción. Para Gómez-Ramírez et al. (25), esta metodología está basada en el modelo de aprendizaje experiencial de Dewey: la construcción de conocimiento a partir de una experiencia concreta se representa como un proceso cíclico, en el que las distintas fases (experiencia concreta, reflexión, conceptualización y aplicación) están interrelacionadas. En este sentido, no basta con una experiencia para provocar conocimiento, sino que para que esto ocurra realmente resultan necesarias la participación y la implicación cognitiva del sujeto, buscando sentido sobre lo experimentado, relacionándolo con su conocimiento previo y desarrollando estructuras conceptuales que le permitan aplicar el nuevo conocimiento a diversas situaciones. Este método es preciso para el tipo de población del estudio en cuestión, ya que se trata de adultos mayores en los cuales, como lo confirman Acero et al. (28), el aprendizaje es un proceso complejo, compuesto por una serie de factores esenciales para su concreción. Señalan que, para que este aprendizaje sea efectivo, se deberían contemplar al menos tres áreas: cognitiva, afectiva y psicomotora, Así mismo, este proceso educativo debe ser alineado con el contexto, los rápidos cambios socioculturales y las necesidades emergentes. Por consiguiente, es válido afirmar que esta herramienta metodológica es la indicada, ya que permite el autodescubrimiento basado en los hechos y fundamentado en la interrelación entre la experiencia y la reflexión. Este tipo de aprendizaje motiva a la formación de las personas involucradas, pues realza sus características de liderazgo y la toma de decisiones, lo que se convierte un elemento esencial y sinérgico que se fortalece en su relación con los demás individuos.

Finalmente, este estudio permite reconocer la utilidad del programa de paciente experto en el proceso de educación que direcciona el profesional de enfermería como una herramienta complementaria para promover el autocuidado. En la medida en que estén más informados, expresen sus necesidades, sean capaces de tomar decisiones e incrementen su autoeficacia, los pacientes adoptarán un rol activo y corresponsable que favorece la reducción de complicaciones y reingresos hospitalarios.

Las instituciones de salud deben estar más abiertas a la inclusión de estos programas, pues, al contar con pacientes más informados y responsables, la eficacia se favorece significativamente. De esta manera, se transforma el cuidado proporcionado, enmarcándose en un modelo deliberativo y relacional por parte de los profesionales, fundamentado en escucha activa, mayor grado de participación por parte del paciente y consenso en las actuaciones.

\section{Limitaciones}

En vista de que se trata de un estudio piloto, aún no es posible generalizar los datos. Se continuará evaluando este programa a largo plazo y con un número mayor de pacientes. 


\section{CONCLUSIONES}

El Programa del paciente experto en insuficiencia cardiaca se constituye en una intervención costo-efectiva, que pretende proporcionar capacidades para ayudar a otros pacientes a adquirir autoconfianza y habilidades para manejar su condición de salud. El efecto positivo del programa en los conocimientos y satisfacción se elucida debido a que los pacientes se sienten identificados con otros individuos que han vivido la enfermedad. Se combinan, en resumen, los cuidados ofrecidos por los profesionales basados en la evidencia con los de la vivencia y la experiencia.

\section{Financiación}

Convocatoria Interna 2017. Apoyo a semilleros de investigación. Vicerrectoría de Investigación, Pontificia Universidad Javeriana.

\section{REFERENCIAS}

1. Gómez E. Introducción, epidemiología de la falla cardiaca e historias de las clínicas de falla cardiaca en Colombia. Rev Colomb Cardiol. 2016;23:6-12. https://doi.org/10.1016/j.rccar.2016.01.004

2. Tamayo DC, Rodríguez VA, Rojas MX, Rincón M, Franco M, Ibarra MT, et al. Costos ambulatorios y hospitalarios de la falla cardiaca en dos hospitales de Bogotá. $\begin{array}{lll}\text { Acta } & \text { Med Colomb. 2013;38(4):208-212. }\end{array}$ http://actamedicacolombiana.com/ojs/index.php/actamed/article/view/205

3. Díaz R, Díaz J, Fuenmayor-Ojeda V, Parejo JA. Insuficiencia cardíaca aguda: análisis clínico epidemiológico. Med Interna. 2018;34(4):224-236. http://www.svmi.web.ve/ojs/index.php/medint/article/view/491

4. León-González R, García-Esquinas E, Paredes-Galán E, Ferro-Martínez Al, González-Guerrero JL, Hornillos-Calvo M, et al. Alfabetización en salud y resultados de salud en pacientes muy ancianos con insuficiencia cardiaca. Rev Esp Cardiol. 2018; 71(3):178-184. https://doi.org/10.1016/j.recesp.2017.05.029

5. Contel JC. La atención integrada y el reto de la cronicidad. Enferm Clin. 2018; 28(1):1-4. https://doi.org/10.1016/j.enfcli.2017.12.002

6. Millaruelo-Trillo JM. Importancia de la implicación del paciente en el autocontrol de su enfermedad: paciente experto. Importancia de las nuevas tecnologías como soporte al paciente autónomo. Aten Primaria. 2010;42 Supl 1:41-47. https://doi.org/10.1016/S0212-6567(10)70007-X

7. De Paz-Ranz S. Escuela de familias y pacientes en una unidad de ictus. Rev. Astur. Ter. Ocu. 2018;13:26-31. https://www.therapeutica.es/index.php/numerospublicados/ultimo-numero/item/download/245_eea4837c87f816a9a3062c07f0e15095

8. Lorig K. Chronic Disease Self-Management Program: Insights from the Eye of the Storm. Front Public Health. 2015;3:153. Artículo corregido y publicado de Front Public Health. 2014;2:253. https://doi.org/10.3389/fpubh.2015.00153

9. González-Mestre A. Autonomía del paciente con enfermedades crónicas: de paciente pasivo a paciente activo. Enferm Clin. 2013;24(1):1-98. https://doi.org/10.1016/j.enfcli.2013.11.005

10. Griffiths C, Foster G, Ramsay J, Eldridge S, Taylor S. How effective are expert patient (lay led) education programmes for chronic disease?. BMJ. 2007; 334(7606):1254-1256. https://doi.org/10.1136/bmj.39227.698785.47

11. Holden RJ, Schubert CC, Mickelson RS. The patient work system: An analysis of self-care performance barriers among elderly heart failure patients and their informal 
caregivers.

Appl

Ergon.

2015;47:133-150.

https://doi.org/10.1016/j.apergo.2014.09.009

12. Ministerio de la Protección Social. Buenas Prácticas para la Seguridad del Paciente en la Atención en Salud. Bogotá: Ministerio; 2010. http://www.acreditacionensalud.org.co/ea/Documents/Guia\%20Buenas\%20Practicas \%20Seguridad\%20del\%20Paciente.pdf

13. Badia Rafecas, W., et al. ¿El programa Paciente Experto Catalunya ${ }^{\circledR}$ mejora la adherencia al tratamiento anticoagulante oral?. Revista ROL de Enfermería. 2018;41(3): 210-216. https://medes.com/publication/134752

14. Miro $O$, Gil V, Javaloyes $O$, Llorens P. Acerca de las guías europeas de insuficiencia cardiaca de 2016. Rev Clin Esp. 2018;218(3):158-159. https://doi.org/10.1016/j.rce.2017.10.008

15. Hall JA, Dornan MC. Meta-analysis of satisfaction with medical care: Description of research domanin and analysis of overall satisfaction levels. Soc Scie Med. 1988; 27(6):935-939. https://doi.org/10.1016/0277-9536(88)90012-3

16. Molano D. Carolina H. Papel de la enfermería en las unidades de falla cardiaca y educación en falla cardiaca, Rev Colomb Cardiol. 2016;23(S1):31-33. http://dx.doi.org/10.1016/j.rccar.2016.01.009

17. Salvadó-Hernández C, Cosculluela-Torres $P$, Blanes-Monllor $C$, Parellada-Esquius $\mathrm{N}$, Méndez-Galeano C, Maroto-Villanova N, et al. Insuficiencia cardiaca en atención primaria: actitudes, conocimientos y autocuidado. Aten Primaria. 2018;50(4): 213-221. https://doi.org/10.1016/j.aprim.2017.03.008

18. Barrios K. El conocimiento libre para la transformación universitaria. Congreso Iberoamericano de Ciencia, Tecnología, Innovación y Educación; 2014 Nov 12-14. Buenos Aires: Organización de Estados Iberoamericanos (OEI); 2015. p 1-11. https://www.oei.es/historico/congreso2014/memorias2014.php 19. Molina-Escribano $\mathrm{F}$, Castaño-Moreno E, Massó-Orozco J. Experiencia Educativa con un Paciente Experto. Rev Clin Med Fam. 2008;2(4):178-180. http://www.revclinmedfam.com/articulo.php?art=11

20. González Assumpció Mestre. La autonomía del paciente con enfermedades crónicas: De paciente pasivo a paciente activo. Enferm Clin. 2014;24(1):67. https://doi.org/10.1016/j.enfcli.2013.11.005

21. Barreiro M, Velasco E, Renilla A, Torres F, Martín M, De la Hera JM. Grado de conocimiento sobre su enfermedad cardiaca entre los pacientes hospitalizados. Rev Esp Cardiol. 2013;66(3):229-30. https://doi.org/10.1016/j.recesp.2012.07.022

22. Pesquera-Cabezas R, López-López LM. Un ejemplo innovador en políticas de salud. Gac Sanit. 2016;30(2):158-159. https://doi.org/10.1016/j.gaceta.2015.11.008 23. Torres-Gonzáles GC, León-Manco RA. Nivel de satisfacción de los pacientes atendidos en el Servicio de Ortodoncia de una Clínica Dental Docente peruana. Revista Estomatol Herediana. 2015;25(2):122-132. https://doi.org/10.20453/reh.v25i2.2458

24. Santana de Freitas J, Bauer de Camargo Silva AE, Minamisava R, Queiroz Becerra AL, Gomes de Sousa MR. Calidad de los cuidados de enfermería y satisfacción del paciente atendido en un hospital de enseñanza. Rev. Latino-Am. Enfermagem. 2014;22(3):454-460. https://doi.org/10.1590/0104-1169.3241.2437

25. Gómez-Ramírez OJ, Carrillo González GM, Cárdenas DC. Encuesta de satisfacción con el cuidado de la salud en las personas con enfermedad crónica. Enferm. glob. 2016;15(4):321-330. https://doi.org/10.6018/eglobal.15.4.226851

26. Ampuero CE. Calidad del servicio y fidelización del paciente en la clínica oftálmica instituto de la visión en el distrito de San Borja 2016 [Tesis de pregrado]. Lima: Universidad Inca Garcilaso de la Vega; 2016: p. 29-31. http://repositorio.uigv.edu.pe/handle/20.500.11818/1714 
27. Diaz M, Gil F. Experiencia con una Nueva Metodología de Enseñanza: Aprendizaje Preferencial Complementario. España: Universidad de Las Palmas de Gran Canaria; 2010: p. 284.285. http://www2.dis.ulpgc.es/ mdiaz/ExperienciaAPC.pdf 28. Acero-Pereira C, Hidalgo MV, Jiménez L. Adult Learning Processes in Non-Formal Education Contexts. Universitas Psychologica. 2018;17(2):155-164. https://doi.org/10.11144/Javeriana.upsy.17-2.paac 